\title{
LA BIBLIA VISIGÓTICA DE LA CATEDRAL DE LEÓN (CÓDICE 6): PRIMEROS APUNTES PARA UN ESTUDIO ARQUEOLÓGICO ${ }^{1}$
}

\author{
Ana SUÁREZ GONZÁLEZ \\ Universidade de Santiago de Compostela
}

\begin{abstract}
RESUMEN: La Biblia visigótica del Archivo de la Catedral de León (Códice 6) es la llamada Biblia de Albares o Biblia de 920, por su supuesto origen geográfico y cronológico. No es un ejemplar desconocido, al contrario, pero son muchas las incógnitas que aún plantea. Su texto, especialmente una pieza hagiográfica añadida (la Vida de San Froilán) ha despertado el interés de los eruditos y su peculiar iluminación ha merecido la atención de los historiadores del Arte. Sin embargo, ni la materialidad del manuscrito ni su escritura han sido objeto de un estudio pormenorizado. Como introducción a una aproximación desde la "arqueología del libro", se lleva a cabo un primer acercamiento a caracteres externos del códice: los materiales y el resultado de las llamadas "tareas preparatorias" para la escritura y la iluminación.
\end{abstract}

PALABRAS CLAVE: Arqueología del libro. Biblia de 920. Catedral de León. Escritura visigótica. Siglo $\mathrm{X}$.

ABSTRACT: The Visigothic Bible (Leon, Archivo de la catedral, cod. 6) is also knows as the Bible of Juan and Vimara, the Bible of 920, or the Albares Bible, according to its authors, date and alleged precedence. Although it is a famous codex, it still raises important questions. Both its text, to which a Vita of Saint Foila was added, and its peculiar illumination attracted the interest of Historians and Art Historians; nevertheless neither its materiality nor its scripture were seriously approached. In this paper I will focus on the "archaeology" of the book, i.e. on pattern which lies behind the preparation of its writing surface.

KEYWORDS: Book archeology. Bible of 920. León Cathedral. Visigothic scripture. Tenth century.

El Códice 6 del Archivo de la Catedral de León ${ }^{2}$, hoy pieza relevante del Museo catedralicio $^{3}$, es la llamada Biblia de Juan y Vimara -artífices mencionados en

${ }^{1}$ Recibido el 14 de septiembre de 2011. Aceptado en el consejo de 8 de noviembre de 2011.

2 Este trabajo no habría sido posible sin la colaboración de D. Manuel Pérez Recio, canónigo archivero, y del Cabildo de la Catedral de León.

${ }^{3}$ Debido a esta circunstancia, ha sido necesario realizar la mayor parte del estudio en el archivo capitular a partir de reproducciones. El Cabildo de la Catedral de León autorizó la consulta directa del volumen el 13 de septiembre de 2011, lo que permitió abordar aspectos de la materialidad que sólo es 
diversas suscripciones ${ }^{4}$-, Biblia del abad Mauro -personaje que figura en un ex libris laberíntico coetáneo ${ }^{5}$ - y Biblia de Albares o Biblia de 920, por su supuesto origen geográfico y cronológico. De estas denominaciones podría deducirse que nos hallamos ante un ejemplar de contextualización segura, pero no es así. Debido, parece, al uso inapropiado de reactivos para avivar la escritura, ha desaparecido el colofón que se disponía en la primera columna de la última página ${ }^{6}$, daño ya evidente a finales del siglo XIX, como advierte explícitamente S. Berger ${ }^{7}$ y se deduce de las observaciones de J. E. Díaz Jiménez y R. Beer en el primer Catálogo publicado de los códices catedralicios ${ }^{8}$.

La lectura de esta nota conclusiva que llevó a cabo M. Risco ${ }^{9}$ ha servido de base a investigadores y eruditos posteriores, que, corrigiendo y/o completando la interpretación del sabio agustino ${ }^{10}$, han adscrito la Biblia al cenobio de Albares y

posible analizar a partir del original, pero, obviamente, no fue posible en tan corto tiempo llevar a cabo un estudio arqueológico pormenorizado. De ahí el título de este trabajo. Cualquier investigador familiarizado con la "arqueología del libro" echará en falta, entre otros aspectos, una descripción detallada de la mise en page (sistema de pinchado y de pautado, descripción de la configuración de página en un número relevante de folios, etc.). Asimismo, ateniéndome a la brevedad impuesta a las "Notas científicas", reservo para otra ocasión el análisis de la escritura.

${ }^{4}$ Suscripciones que se hallan en los ff. 2r (Vimara, en laberinto), 91v (Juan), 202r (Juan), 211r (Juan), 216r (Juan), 217r (Juan) y 233v (Vimara). Editadas, entre otros, por J. E. DíAZ JIMÉNEZ .- R. BEER (1888). Noticias bibliográficas y catálogo de los códices de la Santa Iglesia Catedral de León. León: Mariano Garzo. pp. 5-6 y Z. GarCía ViLladA (1919). Catálogo de los códices y documentos de la catedral de León. Madrid: Imprenta clásica española, pp. 36-37.

${ }^{5}$ En el f.2r. La leyenda "Maurus abbati librum" se dispone en la mitad superior y la suscripción "Vimara presbiter fecit" en la inferior.

${ }^{6}$ En el f.275v. En la mitad derecha de la misma página se hallan dos laberintos. De uno sólo se conservan algunas letras y el otro permanece con los espacios destinados a escritura en blanco.

${ }^{7}$ S. Berger (1893). Histoire de la Vulgate pendant les premiers siècles du Moyen Âge. Paris: Hachette, p.18.

${ }^{8}$ J. E. DíAZ JiMÉNEZ .- R. BEer (1888). Noticias bibliográficas, pp. 5-8. Aunque no lo advierte explícitamente, la entrada referida a la Biblia en el catálogo más conocido (Z. GARCía VILLADA (1919). Catálogo, pp. 36-37) reproduce en buena parte las noticias recogidas en el instrumento descriptivo de 1888.

9 M. RISCO (1784). España sagrada. Tomo XXXIV. Madrid: Pedro Marín, p.165 y M. RISCO (1792) Iglesia de León, y monasterios antiguos y modernos de la misma ciudad. Madrid: Blas Román, p.78: "In monasterio vocabulo Alb."

${ }^{10}$ M. Gómez Moreno (1925). Catálogo monumental de España. Provincia de León (1906-1908). Madrid: Ministerio de Instrucción Pública y Bellas Artes, p.153. Basándose principalmente en la lectura de Risco, no menciona el cenobio de Albares y formula otras hipótesis sobre el origen geográfico. 
fijado su conclusión en el año $920^{11}$. Lamentablemente, hoy no es posible verificar esta información y, por ello, aun admitiendo la solvencia y responsabilidad de cuantos en su momento pudieron acceder total o parcialmente al referido colofón, prefiero designar el manuscrito Biblia visigótica de la catedral de León, tomando en consideración dos realidades incuestionables: su tipo de escritura -visigótica redonda o sentada- y el único dato indiscutible de su historia: el lugar en el que se encuentra actualmente.

\section{UNA BIBLIA INCOMPLETA, ILUMINADA, ESTIMADA Y ¿ESTU- DIADA?}

Pese a su denominación, el Códice 6 del archivo catedralicio no contiene la Sagrada Escritura completa. Se trata, probablemente, de la segunda parte de una biblia dividida oginariamente en, al menos, dos volúmenes.

Por lo que se refiere al texto originario, recoge, acompañados de diversas piezas introductorias y/o complementarias (sumarios, prólogos, etc.), la mayor parte habituales en los manuscritos bíblicos medievales, los libros proféticos (Is.Mal.) $)^{12}$, Iob, Tob., Esdr., Neh., Esth., Iudith, 1-2 Mach., Mt., Mc., Lc, Io., seguidos -tras un conjunto de opúsculos ${ }^{13}$ - de Act., las epístolas paulinas (Rom. - Hebr.), las católicas (Iac.- Iudae), Act. y Apoc. En el f.101r-v, aprovechando página y media en blanco, entre los libros de Job y Tobías ${ }^{14}$, se añadió posteriormente una breve

11 J. M. DE EgUREN (1859). Memoria descriptiva de los códices notables consevados en los archivos eclesiásticos de España. Madrid: M. Rivadeneyra, p.46. A diferencia de otros autores, incluye en la transcripción, completo, el vocablo "Albares". La propuesta de un cenobio sito en Albares, localidad berciana en el valle del Boeza, como origen de esta Biblia se debe a A. Quintana, quien, además, plantea que el abad Mauro mencionado en el colofón pudo ser el prelado de otro monasterio del Bierzo: Santa Cruz de Montes (A. QuinTANA PRIETO (1972). «Santa María de Albares y su escritorium». Yermo. Cuadernos de Historia y de espiritualidad monástica, X.1, pp. 67-105).

${ }^{12}$ Isaías se inicia en el f.5r, precedido del prólogo "Nemo cum prophetas...ecclesiis eius diutius exultarent" que el epígrafe inicial -en el f.4v- atribuye, como es habitual, a san Jerónimo.

${ }^{13}$ En los ff. 202r-217v. Breves exposiciones sobre cada uno de los evangelios (ff. 202r-216r), el opúsculo que abre el epígrafe INCIPIT ORTUM ET OBITUM APOSTOLORUM (f.216r-v) y el tratadillo encabezado por INCIPIT DE GENEALOGIIS (ff. 216v-217r). El f.217v permanece en blanco.

${ }^{14} \mathrm{Job}$ concluye al final de la primera columna del f.101r, folio en el que hay un orificio ovalado de gran tamaño que afecta a la columna de texto más próxima al canal. Tal vez no se consideró apropiado iniciar bajo este orificio -que inutiliza siete rectoras- un nuevo libro bíblico y se prefirió dar comienzo al prefacio al libro de Tobías en un recto sin tachas (f.102r). 
pieza hagiográfica que ha recibido especial atención en la literatura científica: la Vida de san Froilán ${ }^{15}$.

Es relevante la iluminación del libro, concentrada especialmente en la escritura decorativa, jerarquizada y de factura cuidada, que abunda a lo largo del volumen. Abren el códice, a toda página, una cruz -"cruz de Oviedo"- (f.1v) ${ }^{16}$, el laberinto que recoge el ya citado ex libris y la suscripción de uno de los copistas (f.2r) ${ }^{17}$, la rosa de los vientos (f.3r) y un cuadro conformado por treinta y seis círculos que cobijan figuras humanas y animales reales y fantásticos (f.3v). En los ff. 149r-155v se hallan arcos sobre columnas destinados a cobijar los "cánones de concordancias"18 con los correspondientes símbolos de los evangelistas (ff.149v-

\footnotetext{
${ }^{15}$ Pieza que destaca el P. Ibarreta en un informe o "catálogo" (Santo Domingo de Silos, Archivo del monasterio, Catálogo $\mathrm{D}^{2}$ (olim leg. 110) redactado entre 1770-1771 (M. C. Vivancos GómeZ (1996). Glosas y notas marginales de los manuscritos visigóticos del monasterio de Santo Domingo de Silos. Santo Domingo de Silos: Abadía de Silos, p.421) y en el que dedica una entrada a esta biblia. Afirma que "corrige en mucha parte la que anda en los brebiarios". Las características de la escritura, también visigótica, pero desmañada y con rasgos cursivos, denotan claramente que se trata de una adición posterior. M. Risco se pronuncia contradictoriamente sobre la incorporación de esta pieza: "el escritor fue el mismo, que el del Códice como lo indica la forma de la letra, aunque es algo más abultada que la del texto sagrado, y la tinta más negra" (M. RISCO (1784). España sagrada. Tomo XXXIV. Madrid: Pedro Marín, p.165), "se escribió después la vida del glorioso san Froylan, Obispo de Leon. La forma de la letra es algo mas abultada que la del Códice, y la tinta mas negra; pero reconocida esta leyenda por varones doctos en la Polygraphia Española, se ha tenido por cierto que esta historia se escribió en el mismo tiempo que el Códice, y por un mismo escritor" (M. RISCO (1792). Iglesia de León, y monasterios antiguos y modernos de la misma ciudad. Madrid: Blas Román, p.79). Más atinadas son las observaciones al respecto de J. E. Díaz Jiménez y R. Beer: "Entre los libros de Job y de Tobías, en una columna, que el primer copista dejó el blanco (fol. 1rb), se halla escrita, también en letra gótica, la vida de San Froilán, patrón de León. (...) Asimismo lleva la subscripción Joannes Diaconus scripsit, pero creemos que es una imitación, queriendo el segundo copista identificarse con el primero, tan notable" (J. E. DíAZ JIMÉNEZ .- R. BEER (1888). Noticias bibliográficas, p.7). Si bien en el Catálogo del fondo capitular Z. García Villada sólo señala que el opúsculo se ha realizado en "letra un poco mayor" (Z. GARCía ViLLADA (1919). Catálogo, p.36), en su tratado de Paleografía indica que el texto es obra de un copista posterior (Z. GARCIA VILladA (1923). Paleografía española precedida de una introducción sobre la paleografía latina. I. Texto. Madrid: Publicaciones de la Revista de Filología Española, p.171. Pese a la evidencia gráfica, afirma A. Quintana que esta "biografía" de san Froilán es obra de "Juan diácono, el autor del códice que nos ocupa" (A. QUINTANA PRIETO (1972). "Santa María de Albares y su escritorium", p.73), opinión que comparten otros investigadores (V. GARCÍA LOBO (2002). «Centro de cultura escrita». En La catedral de León. Mil años de historia. León: Edilesa, p.309).

${ }^{16}$ El f.1r se halla en blanco.

${ }^{17}$ El vuelto del folio permanece en blanco.

18 A. ViÑAYO GONZÁLEZ (1990), «Diácono Juan ayudado por el presbítero Vimara». En Las Edades del Hombre. Libros y documentos en la Iglesia de Castilla y León. Valladolid, p.54.
} 
$154 v)^{19}$. En el f.201v, ocupando la práctica totalidad del espacio reservado a la segunda columna de texto $^{20}$, se sitúan cuatro escenas neotestamentarias acompañadas de explanationes-: Anunciación, María y Jesús, curación del ciego y encuentro de Jesús con la samaritana ${ }^{21}$. Representaciones simbólicas de los evangelistas -identificadas mediante tituli en caracteres publicitarios- encabezan breves tratados relativos a los cuatro evangelios ${ }^{22}$. Salvo la de Juan (f.214r) ${ }^{23}$, se disponen a toda página: Mateo (f.202r), Marcos (f.209r) y Lucas (f. 211r). En el margen de canal del f.209v aparece nuevamente, iniciando el tratadillo correspondiente al evangelio de Mateo, el león. La última página del códice recoge, en marcos ornados, el maltrecho colofón y dos laberintos, uno de ellos inconcluso.

Calificado de "excelente" por M. Risco ${ }^{24}$ y considerado "uno de los más notables que se han escrito en España" por J. M. de Eguren ${ }^{25}$, el códice parece, a primera vista, sobradamente conocido ${ }^{26}$. Sin embargo, es evidente que, pese al

19 Tal vez debido a un error de cálculo, a una reproducción irreflexiva del exemplar o a otras razones, los espacios bajo los arcos destinados a recibir texto permanecen en blanco en los ff. 149r y 155r-v.

${ }^{20}$ El marco, de 265 x $80 \mathrm{~mm}$, no se ciñe estrictamente a la segunda columna pautada.

${ }^{21}$ Son las siguientes: "Maria cum Gabriel", "Maria cum Ihesu", "Ubi Ihesus inluminat cecum", "Ubi Ihesus loqvitur cum mulier samaritana", "puteum". Sobre la relación de estas representaciones y su disposición (en registros superpuestos) con otras manifestaciones artísticas, J. WiLliams (1999). "The Bible in Spain". En Imaging Early Medieval Bible. University Park: The Pennsylvania State University Press, pp. 182-185 y G. FernÁNDEZ SOMOZA (2004). «La Biblia de León del año 920 en el contexto de la miniatura hispánica». En La catedral de León en la Edad Media. León: Universidad de León, pp. 502, 506-507.

${ }^{22} \mathrm{Se}$ ha supuesto erróneamente que estas representaciones simbólicas de cada uno de los evangelistas dan "paso a su evangelio"(G. FERNÁNDEZ SOMOZA (2004). «La Biblia de León», p.502).

${ }^{23}$ En la mitad inferior de la página. La representación es mucho menor y más sencilla que las restantes. La primera columna del vuelto del folio permanece en blanco. Tal vez la ubicación de la ilustración responda a un error de cálculo en el traslado del texto previo o del siguiente.

${ }^{24}$ M. RisCo (1784). España sagrada. Tomo XXXIV. Madrid: Pedro Marín, p.165.

25 J. M. DE EGuREn (1959). Memoria descriptiva, p.46. En opinión de J. E. DíAz JiMÉNEZ.- R. BEER, Notas bibliográficas, p.XXIV, nota 1 , no consultó directamente el ejemplar y ni siquiera hay constancia de su estancia en León.

${ }^{26}$ Para establecer un primer "estado de la cuestión" pueden tenerse en cuenta las relaciones bibliográficas que se recogen en T. AYuso (1953). La Vetus Latina Hispana. Origen, dependencia, derivaciones, valor e influjo universal. Reconstrucción, sistematización y análisis de sus diversos elementos. Coordinación y edición crítica de su texto. I. Prolegómenos (Introducción general, estudio y análisis de las fuentes). Madrid: Consejo Superior de Investigaciones Científicas, p.354, M. C. DíAZ Y DíAZ (1983). Códices visigóticos en la monarquía leonesa. León: Centro de Estudios e Investigación San Isidoro, p.307, nota 24, A. VIÑAYO GoNZÁLEZ (1990), «Diácono Juan ayudado por el presbítero Vimara». En Las Edades del Hombre. Libros y documentos en la Iglesia de Castilla y León. Valladolid, p.54, A. Millares Carlo (M. C. Díaz Y Díaz, A. M. Mundó, J. M. Ruiz Asencio, 
interés suscitado, aspectos relativos a su génesis -contexto, artífices, proceso de confección- y a su materialidad aún no han sido suficientemente abordados. No faltan acercamientos al Legionense ${ }^{1}$ en estudios sobre el texto bíblico transmitido en las biblias medievales ${ }^{27}$ y en los tratados de Paleografía ${ }^{28}$. Asimismo, destacan, por su frecuencia, las aproximaciones desde la Historia del Arte ${ }^{29}$ que subsanan la carencia de análisis subrayada por M. Gómez Moreno hace un siglo: "No obstante la grandísima importancia de este códice, son deficientes por demás las descripciones suyas que conozco, sobre todo en lo que se refiere a la parte artística" ${ }^{30}$. En resumen, de esta biblia "sobresaliente" se han abordado, en mayor o menor medida, los aspectos "sobresalientes": el texto que guarda y la iluminación inusual- que la embellece, pero apenas se ha escrito nada sobre ella como objeto artesanal fruto de empleo de determinados materiales y del desarrollo de técnicas específicas, medios y procedimientos que, una vez analizados y puestos en relación con la información proporcionada por el texto, la iluminación y la escritura, permitirán resolver algunas de las incógnitas que presenta y evitarán que se perpetúen errores fruto de un acercamiento superficial o parcial al códice. Sirvan estos apuntes referidos a los materiales y al resultado de las llamadas "tareas preparatorias" para la escritura y la iluminación de introducción al estudio arqueológico completo del que la Biblia carece y que, ojalá, pueda llevarse a cabo en algún momento.

\section{SOPORTE, DIMENSIONES, ORGANIZACIÓN DEL VOLUMEN.}

El códice bíblico ha llegado a nosotros en buen estado de conservación. Tras dos folios de papel incorporados tardíamente, se suceden 277 folios en pergamino.

B. Casado Quintanilla, E. Lecuona Ribot (eds.) (1999), Corpus de Códices visigóticos, I, Estudio. Las Palmas de Gran Canaria: Fundación de Enseñanza Superior a Distancia de Las Palmas de Gran Canaria, pp. 68-69 y M. SÁNCHEZ MARIANA (2003). «Fuentes literarias para la catalogación y estudio de los códices de la catedral de León». En León y su historia. Miscelánea histórica. VIII. León: Centro de Estudios e Investigación San Isidoro, pp.356-360.

${ }^{27}$ S. BERGER (1893). Histoire de la Vulgate pendant les premiers siècles du Moyen Âge. Paris: Hachette, pp. 17-18, T. AYUSO (1953). «La Vetus Latina Hispana», pp. 353-354.

${ }^{28}$ Z. Garcia Villada (1923). Paleografía española, pp. 170-171.

${ }^{29}$ A modo de ejemplo, J. DomínguEZ Bordona (1962), «Miniatura». En Ars Hispaniae. Historia Universal del Arte Hispánico, XVIIII. Madrid: Plus ultra, p.21, J. YARZA LuACES (1994). «La miniatura altomedieval». Historia del Arte de Castilla y León. Tomo I. Prehistoria, Edad Antigua y Arte prerrománico. Valladolid: Ámbito, pp. 247-248, J. Williams (1999). «The Bible in Spain». En Imaging Early Medieval Bible. University Park: The Pennsylvania State University Press, pp. 182-185, F. GALVÁN FREILE (2002). «Los manuscritos iluminados». En La catedral de León. Mil años de historia. León: Edilesa, p.315 y G. FERNÁNDEZ SOMOZA (2004). «La Biblia de León», pp. 409-507.

\footnotetext{
${ }^{30}$ M. GÓMEZ MORENo (1925). Catálogo monumental, p.153.
} 
Debido a dos omisiones en una foliación relativamente reciente ${ }^{31}$, el último folio aparece signado con el número $275^{32}$. Pese al error ${ }^{33}$, y con el fin de no dificultar las referencias, será la numeración empleada en este trabajo.

Los folios miden $365-375 \times 230-245 \mathrm{~mm}^{34}$. Es evidente, sin embargo, que las dimensiones originarias fueron mayores y que los márgenes se han recortado. Como consecuencia, elementos auxiliares -el titulus currens $^{35}$, perforaciones maestras para guiar el pautado, reclamos ${ }^{36}$ - y motivos ornamentales empleados en iniciales decoradas que se sitúan en el primer renglón de texto ${ }^{37}$ presentan mutilaciones.

El pergamino es muy heterogéneo; varía el grosor, la flexibilidad, la densidad de los restos de folículos pilosos en el lado pelo, el color, etc. Predomina el de color marfileño y el ligeramente amarillento, con cara de la carne que se distingue de la pars pili por ser más clara, si bien no faltan los casos en los que la diferencia entre ambas faces resulta casi imperceptible. El empleo de pieles defectuosas y su aprovechamiento extremo afean el códice. Son numerosos los folios con tachas. Abundan los orificios circulares y ovales -resultado de la tensión ejercida sobre zonas frágiles de la membrana- algunos de los cuales, bien por sus grandes dimensiones, bien por su número en el mismo folio o por su presencia en el interior del cuadro de justificación ${ }^{38}$, obligaron a los copistas a modificar la disposición del texto $^{39}$. Aunque menos numerosos que los orificios, aparecen ojos $\operatorname{traslúcidos}^{40} \mathrm{y}$

\footnotetext{
${ }^{31}$ Realizada a lápiz, en arábigos, se encuentra en el ángulo superior derecho de los rectos.

${ }^{32}$ Razón por la que en las descripciones del libro publicadas hasta la fecha se mencionan sólo 275
} folios.

${ }^{33}$ Se subsanaron los errores signando el folio que sigue al 150 como "150 bis" y el que se sitúa tras el 190 "190bis".

${ }^{34}$ Sirvan de muestra las dimensiones de los ff. 1 (375 x $\left.232 \mathrm{~mm}\right), 4$ (370 x $\left.240 \mathrm{~mm}\right), 7$ (369 x $245 \mathrm{~mm}), 20(370 \times 238 \mathrm{~mm}), 99(367$ x $241 \mathrm{~mm}), 100(368$ x $242 \mathrm{~mm}), 136$ (365 x $240 \mathrm{~mm}), 203$ $(369$ x $239 \mathrm{~mm}), 218(368 \times 237 \mathrm{~mm}), 254(371 \times 234 \mathrm{~mm})$ y $258(371$ x $239 \mathrm{~mm})$.

35 Es relativamente frecuente a lo largo del volumen la mutilación o la desaparición total del titulus currens por esta razón, si bien en otros casos la ausencia de este elemento de referencia se explica por otros motivos, como el contenido de las páginas.

${ }^{36}$ Cercenado el reclamo del f.209v.

${ }^{37}$ Ejemplos en los ff. 80v, 180v, 249v y 264r.

${ }^{38}$ Orificios en el interior del cuadro de justificación de los ff. 24, 26, 27, 32, 52, 73, 84, 100, 101, 111, 146, 161, 162, 169, 176, 189, 190, 207, 224, 229, 233, 245, 252, 254, 255, 256, 258, 272 у 273.

39 Orificios en el margen superior de los ff. 38, 119, 252, 267 y 274. En el margen inferior de los ff. $51,131,139,140,144,145,181,246,253,254$ y 255 , en el margen de pliegue de los ff. 20, 22, $26,133,134,166,253$ y 256 y en el margen de canal de los ff. 7, 28, 31, 36, 61, 62, 71, 75, 77, 95, 
desgarros reparados ya en el momento de fabricación mediante zurcidos $^{41}$. Pese al recorte de los márgenes, la orilla de la piel, señal del aprovechamiento total de las hojas, resulta visible en ciertos folios ${ }^{42}$.

Los folios se distribuyen en treinta y cuatro cuadernos. Excepto el primero, facticio, todos se inician y concluyen con páginas correspondientes a la cara pelo del pergamino y, salvo en dos casos -que advertiré-, se verifica la Regla de Gregory en la disposición de los bifolios y folios sueltos de cada fascículo ${ }^{43}$.

El primer cuaderno, facticio, consta de sólo tres folios. El f.1r corresponde al pelo del pergamino y el $3 \mathrm{v}$ a la carne ${ }^{44}$.

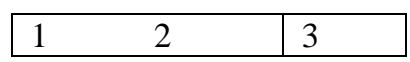

Siguen quince cuaterniones completos ${ }^{45}$.

\begin{tabular}{|c|c|c|c|c|c|c|c|}
\hline 4 & 5 & 6 & 7 & 8 & 9 & 10 & 11 \\
\hline 12 & 13 & 14 & 15 & 16 & 17 & 18 & 19 \\
\hline 20 & 21 & 22 & 23 & 24 & 25 & 26 & 27 \\
\hline 28 & 29 & 30 & 31 & 32 & 33 & 34 & 35 \\
\hline 36 & 37 & 38 & 39 & 40 & 41 & 42 & 43 \\
\hline 44 & 45 & 46 & 47 & 48 & 49 & 50 & 51 \\
\hline 52 & 53 & 54 & 55 & 56 & 57 & 58 & 59 \\
\hline 60 & 61 & 62 & 63 & 64 & 65 & 66 & 67 \\
\hline 68 & 69 & 70 & 71 & 72 & 73 & 74 & 75 \\
\hline
\end{tabular}

$99,123,125,128,146,158,189,190,192,193,194,197,199,205,218,219,237,247,254,264$ у 265.

${ }^{40}$ Ejemplos en los ff. 20, 37, 47, 192, 207, 217, 218, 254, 259 у 260.

${ }^{41}$ Ejemplos en los ff. 42, 48 y 196.

${ }^{42}$ En los ff. 209, 245, 247, 273, 274 y 275.

${ }^{43}$ Se adapta el diagrama empleado en F. FERY-Hue (2005). «L'organisation du volume». En Lire le manuscrit médiéval: observer et décrire. Paris: Armand Colin, p.65. La línea quebrada indica la costura del cuaderno.

${ }^{44}$ Se verifica la Ley de Gregory en el interior del fascículo. Una costura adicional para unir los tres folios, el último de los cuales es, sin duda, un folio suelto, encubre la estructura del cuaderno. Pese a la diferencia de color que se advierte entre el f.1r y el f.2v-páginas correspondientes a pelo de la membrana-, los dos folios pueden ser solidarios. No es posible afirmarlo sin despojar al fascículo de esta costura adicional que oculta la línea de pliegue. La cara pelo del f.2 (vuelto) es más clara que la del f.1 (recto), pero no puede descartarse que el mayor oscurecimiento responda a su mayor exposición por tratarse de la página inicial del libro.

45 Teniendo en cuenta que en todos estos cuadernos la primera página corresponde al pelo de la membrana, se rompe la Ley de Gregory entre el f.3v (carne) y el f.4r (pelo). 


\begin{tabular}{|c|c|c|c|c|c|c|c|}
\hline 76 & 77 & 78 & 79 & 80 & 81 & 82 & 83 \\
\hline 84 & 85 & 86 & 87 & 88 & 89 & 90 & 91 \\
\hline 92 & 93 & 94 & 95 & 96 & 97 & 98 & 99 \\
\hline 100 & 101 & 102 & 103 & 104 & 105 & 106 & 107 \\
\hline 108 & 109 & 110 & 111 & 112 & 113 & 114 & 115 \\
\hline 116 & 117 & 118 & 119 & 120 & 121 & 122 & 123 \\
\hline
\end{tabular}

El decimoséptimo fascículo es un senión.

\begin{tabular}{|l|l|l|l|l|ll|l|l|l|l|l|}
\hline 124 & 125 & 126 & 127 & 128 & 129 & 130 & 131 & 132 & 133 & 134 & 135 \\
\hline
\end{tabular}

El siguiente cuaderno, facticio, consta de trece folios. Los ff. 137-146 son cinco bifolios embutidos y los restantes se incorporaron mediante pestañas ${ }^{46}$. El fascículo se abre, como es habitual, en pars pili de la membrana y se rompe la Ley de Gregory entre los ff. 147v (pelo) y 148r (carne).

\begin{tabular}{|c|c|c|c|c|c|cc|c|c|c|c|c|c|}
\hline 136 & $\mathrm{P}$ & 137 & 138 & 139 & 140 & 141 & 142 & 143 & 144 & 145 & 146 & 147 & $\begin{array}{c}\mathrm{P}+ \\
148\end{array}$ \\
\hline
\end{tabular}

Se disponen a continuación seis cuaterniones completos:

\begin{tabular}{|c|c|c|c|c|c|c|c|}
\hline 149 & 150 & 150bis & 151 & 152 & 153 & 154 & 155 \\
\hline 156 & 157 & 158 & 159 & 160 & 161 & 162 & 163 \\
\hline 164 & 165 & 166 & 167 & 168 & 169 & 170 & 171 \\
\hline 172 & 173 & 174 & 175 & 176 & 177 & 178 & 179 \\
\hline 180 & 181 & 182 & 183 & 184 & 185 & 186 & 187 \\
\hline 188 & 189 & 190 & 190bis & 191 & 192 & 193 & 194 \\
\hline
\end{tabular}

El vigésimoquinto cuaderno comprende siete folios: tres bifolios (195/201, $196 / 199$ y 197/198) y un folio suelto -el 200- incorporado mediante pestaña ${ }^{47}$. Se

\footnotetext{
${ }^{46}$ La sucesión del texto en el cuaderno, sin lagunas, confirma que se trata de pestañas de incorporación de folios y no de talones testigos de componentes desaparecidos. El texto del f.135v continúa en el f.136r. Los ff. 136r-147r ( $1^{\text {a }}$ col. renglón $\left.24^{\circ}\right)$ - corresponden a 1 Mach 13,42-2 Mach 15,40 ). A continuación, ff. $147 \mathrm{r}-148 \mathrm{v}$, diversas piezas introductorias a los evangelios.

${ }^{47}$ No hay salto de texto entre el f.195v y el $196 r$.
} 
inicia y concluye en pelo de la membrana pero se rompe la Ley de Gregory entre el f.200v (pelo) y $201 \mathrm{r}$ (carne) ${ }^{48}$.

\begin{tabular}{|l|l|l|ll|l|l|l|}
\hline 195 & $\mathrm{P}(200)$ & 196 & 197 & 198 & 199 & 200 & 201 \\
\hline
\end{tabular}

A continuación, ocho cuaterniones completos:

\begin{tabular}{|c|c|c|c|c|c|c|c|}
\hline 202 & 203 & 204 & 205 & 206 & 207 & 208 & 209 \\
\hline 210 & 211 & 212 & 213 & 214 & 215 & 216 & 217 \\
\hline 218 & 219 & 220 & 221 & 222 & 223 & 224 & 225 \\
\hline 226 & 227 & 228 & 229 & 230 & 231 & 232 & 233 \\
\hline 234 & 235 & 236 & 237 & 238 & 239 & 240 & 241 \\
\hline 242 & 243 & 244 & 245 & 246 & 247 & 248 & 249 \\
\hline 250 & 251 & 252 & 253 & 254 & 255 & 256 & 257 \\
\hline 258 & 259 & 260 & 261 & 262 & 263 & 264 & 265 \\
\hline
\end{tabular}

Cierra el códice un cuaderno de diez folios con comienzo y conclusión en pars pili. Se rompe la Ley de Gregory entre los ff. 268v (carne) y 269r (pelo) y, lógicamente, entre sus correspondientes solidarios (ff. 273 y 272 respectivamente) $)^{49}$.

\begin{tabular}{|l|l|l|l|ll|l|l|l|l|}
\hline 266 & 267 & 268 & 269 & 270 & 271 & 272 & 273 & 274 & 275 \\
\hline
\end{tabular}

Dos son los recursos coetáneos utilizados para garantizar el orden correcto de los cuadernos: signatura y reclamo. La signatura es el más frecuente, como se advierte en la tabla 1. Ubicadas en el margen inferior de la última página del fascículo en cuestión, se sitúan bajo la primera -la mayor parte ${ }^{50}$ o la segunda columna de texto $^{51}$. Constan de una $Q$ capital $^{52}$ con trazo oblicuo de abreviación cortando el apéndice, seguida de numerales romanos. Son perceptibles ciertas modificaciones en los números, algunos $\operatorname{raspados}^{53} \mathrm{y}$ otros enmendados ${ }^{54} Q \mathrm{y}$

${ }^{48}$ En la primera mitad del cuaderno, la disposición es la siguiente: f.195 (P/C), f.196 (C/P), f.197 (P/C). En la inserción del f.200 (C/P) se tuvo en cuenta, parece, que el f. 199v correspondía a la cara carne de la membrana.

${ }^{49}$ En la primera mitad del cuaderno la sucesión de faces es ésta: f.266 (P/C), f.267 (C/P), f.268 (P/C), f.269 (P/C) y f.270 (C/P).

${ }^{50}$ Signaturas que se sitúan en los ff. $11 \mathrm{v}, 27 \mathrm{v}, 43 \mathrm{v}, 51 \mathrm{v}, 59 \mathrm{v}, 67 \mathrm{v}, 75 \mathrm{v}, 83 \mathrm{v}, 91 \mathrm{v}, 107 \mathrm{v}, 115 \mathrm{v}$, $123 \mathrm{v}, 135 \mathrm{v}, 148 \mathrm{v}, 171 \mathrm{v}, 187 \mathrm{v}, 194 \mathrm{v}, 225 \mathrm{v}, 233 \mathrm{v}, 241 \mathrm{v}, 249 \mathrm{v}$ y $257 \mathrm{v}$.

${ }^{51}$ Las que se hallan en los ff. $19 \mathrm{v}, 35 \mathrm{v}, 99 \mathrm{v}, 163 \mathrm{v}$ y $179 \mathrm{v}$.

52 Inicial de quaternus, quaternio o quaternulus.

${ }^{53}$ Numerales raspados en las signaturas de los ff. $19 \mathrm{v}, 171 \mathrm{v}, 194 \mathrm{v}, 241 \mathrm{v}$ y $249 \mathrm{v}$.

${ }^{54}$ Numeral corregido en la signatura del f.265v. 
numerales pueden aparecer realizados en tinta azul o roja. El empleo de estos colores ya realza las signaturas, pero, además, fueron decoradas con motivos de factura análoga a los que aparecen en las iniciales ornadas que salpican el ejemplar. $\mathrm{Se}$ introducen en elementos fitomorfos ${ }^{55}$ o zoomorfos -principalmente aves y ofidios $^{56}$, excepcionalmente un cuadrúpedo ${ }^{57}$ - realizados asimismo en tinta azul, roja o combinando ambas ${ }^{58}$.

Los reclamos ( $R$ en la tabla 1 ) se disponen horizontalmente en el margen inferior de la última página de diez fascículos, bajo el intercolumnio y muy próximos al borde del folio ${ }^{59}$. Realizados en escritura de módulo muy pequeño y en tinta parda-negra comprenden siempre varias palabras. Sólo en un cuaderno -el $26^{\circ}$, f.209v- es el único recurso empleado para garantizar el orden, en el resto de los fascículos comparten función con las signaturas. Todos tienen correspondencia en el incipit de la página siguiente.

Tabla 1

\begin{tabular}{|c|c|c|c|c|c|}
\hline cuaderno (ff.) & signatura & $R /-$ & cuaderno (ff.) & signatura & reclamo \\
\hline $1^{\circ}($ ff. $1-3)$ & - & - & $18^{\circ}$ (ff. 136-148) & Q XVII & - \\
\hline $2^{\circ}($ ff. $4-11)$ & Q I & - & $19^{\circ}$ (ff. 149-155) & - & - \\
\hline $3^{\circ}$ (ff. 12-19) & $\mathrm{Q}[\ldots]$ & - & $20^{\circ}$ (ff. 156-163) & Q XVIIII & $\mathrm{R}$ \\
\hline $4^{\circ}$ (ff. 20-27) & Q III & - & $21^{\circ}$ (ff. 164-171) & $\mathrm{Q} X X[\ldots]$ & - \\
\hline $5^{\circ}($ ff. $28-35)$ & Q IIII & - & $22^{\circ}$ (ff. 172-179) & $\mathrm{Q} X \mathrm{XI}^{60}$ & - \\
\hline $6^{\circ}($ ff. $36-43)$ & $\mathrm{QV}$ & - & $23^{\circ}$ (ff. $180-187$ ) & Q XXII & - \\
\hline $7^{\circ}($ ff. $44-51)$ & Q VI & - & $24^{\circ}$ (ff. 188-194) & $\mathrm{Q} X X[\ldots]$ & - \\
\hline $8^{\circ}$ (ff. 52-59) & Q VII & - & $25^{\circ}$ (ff. 195-201) & [sólo marco] & - \\
\hline $9^{\circ}$ (ff. 60-67) & Q VIII & $\mathrm{R}$ & $26^{\circ}$ (ff. 202-209) & - & $\mathrm{R}$ \\
\hline $10^{\circ}($ ff. $68-75)$ & Q VIIII & - & $27^{\circ}$ (ff. $210-217$ ) & - & - \\
\hline $11^{\circ}($ ff. $76-83)$ & QX & - & $28^{\circ}$ (ff. $\left.218-225\right)$ & Q XXIIII & - \\
\hline $12^{\circ}$ (ff. 84-91) & Q XI & - & $29^{\circ}$ (ff. 226-233) & OXXV & - \\
\hline $13^{\circ}$ (ff. 92-99) & Q XII & $\mathrm{R}$ & $30^{\circ}$ (ff. 234-241) & $\mathrm{Q} X X V I^{61}$ & - \\
\hline
\end{tabular}

${ }^{55}$ Signaturas de los ff. 19v, 59v, 91v, 99v, 107v, 135v, 233v y 265v. Dos ofidios conforman una figura vegetal en la signatura del f.83v.

${ }^{56}$ Signaturas de los ff. $11 \mathrm{v}, 27 \mathrm{v}, 35 \mathrm{v}, 43 \mathrm{v}, 51 \mathrm{v}, 67 \mathrm{v}, 75 \mathrm{v}, 115 \mathrm{v}, 123 \mathrm{v}, 148 \mathrm{v}, 163 \mathrm{v}, 171 \mathrm{v}, 179 \mathrm{v}$, $194 \mathrm{v}, 225 \mathrm{v}, 241 \mathrm{v}, 249 \mathrm{v}, 157 \mathrm{v}$ y $265 \mathrm{v}$.

${ }^{57}$ Signatura del f.187v.

${ }^{58}$ En el f.201 se ha realizado un marco fitomorfo en tinta azul, pero no se ha introducido ni la inicial ni el numeral.

${ }^{59} \mathrm{El}$ reclamo del f.209v ha desaparecido en buena parte, mútilo por hallarse en el borde inferior del folio. Tal vez el códice contó inicialmente con mayor número de reclamos ahora desaparecidos como resultado del recorte del margen de pie.

${ }^{60}$ Corregido el numeral $I$.

${ }^{61}$ A continuación, carácter eliminado mediante raspado. 


\begin{tabular}{llllll}
$14^{\circ}$ (ff. 100-107) & Q XIII & R & $31^{\circ}$ (ff. 242-249) & Q X [... $]^{62}$ & - \\
$15^{\circ}$ (ff. 108-115) & Q XIIII & R & $32^{\circ}$ (ff. 250-257) & Q XXVII & R \\
$16^{\circ}$ (ff. 116-123) & Q XV & R & $33^{\circ}$ (ff. 258-265) & Q XXVIII ${ }^{63}$ & R \\
$17^{\circ}$ (ff. 124-135) & Q XVI & R & $34^{\circ}$ (ff. 266-275) & - & - \\
\hline
\end{tabular}

\section{CONFIGURACIÓN DE LA PÁGINA.}

Para orientar la ejecución del pautado, manual, se practicaron perforaciones en los folios ${ }^{64}$. Los pinchazos maestros para guiar las líneas verticales se encuentran en los márgenes de cabeza y pie. Los puntos de referencia para el trazado de las rectrices horizontales se hallan en el interior del cuadro de justificación ${ }^{65}$, apreciándose ahora con claridad en el espacio reservado al intercolumnio ${ }^{66}$. Siempre de pequeño tamaño, varía a lo largo del volumen la morfología de estos puntos de referencia. Tienen morfología circular en los cuadernos $2^{\circ}-18^{\circ}$ (ff. 4148 ), $26^{\circ}-27^{\circ}$ (ff. $202-217$ ) y $33^{\circ}-34^{\circ}$ (ff. $258-275$ ) y son planos en los fascículos $19^{\circ}-25^{\circ}$ (ff. $\left.149-201\right)$ y $28^{\circ}-32^{\circ}$ (ff. $\left.218-257\right)^{67}$.

El libro presenta pautado sin color, en surco-relieve o a punta seca ${ }^{68}$. No faltan los folios en los que la intensidad de la incisión ha provocado roturas ${ }^{69}$. La disposición de las perforaciones-guía y la longitud de las directrices -que atraviesan la línea de pliegue- indica que el pautado se llevó a cabo -directa o indirectamente, según los casos- sobre el bifolio desplegado.

${ }^{62}$ Siguen numerales raspados.

${ }^{63}$ Probablemente corregido.

${ }^{64}$ Por las razones señaladas en la nota 3 no ha sido posible en esta ocasión determinar, cuaderno a cuaderno, el sistema de perforación y presentar el correspondiente diagrama.

${ }^{65}$ No siempre coincide el número de perforaciones-guía con el número de directrices realizadas, a veces alguno de los pinchazos extremos no se toma en consideración para la ejecución del entramado, como se advierte en el cuaderno segundo (ff. 4-11). En él se llevaron a cabo 39 puntos de referencia y sólo se realizaron 38 líneas horizontales, la primera picadura no guía rectora alguna.

${ }^{66}$ Debido a las razones expuestas en la nota 3 no es posible por el momento precisar el sistema o sistemas de pautado y presentar los correspondientes diagramas.

${ }^{67}$ Es interesante, para establecer la sucesión en lo que al uso de distintos instrumentos se refiere, la incorporación de un fragmento rectangular de pergamino en cabeza del f.27, con cuatro renglones de texto coetáneo -pero realizado por mano distinta- en recto y vuelto (¿se añadió para suplir un trozo recortado como resultado de una acción correctora? ¿para eliminar un defecto importante en la piel?). El folio presenta pinchazos circulares y el pergamino añadido recibió perforaciones planas.

${ }^{68} \mathrm{La}$ "cata" realizada muestra que el instrumento incidió sobre la cara del pelo, pero no es posible por el momento afirmar que se ha llevado a cabo así la pauta en todos los cuadernos del códice.

${ }^{69}$ En los ff. 230 y 275 
Se realizó un entramado para recibir texto a dos columnas ${ }^{70}$, pero no hay uniformidad, sin embargo, en otros aspectos. Son dos los tipos de pautado. En el primero, perceptible en el segundo fascículo, las dos columnas de texto aparecen delimitadas por doble vertical (pliegue / canal). El segundo, predominante a lo largo del códice, es más sencillo: son sólo cuatro las líneas verticales. En ambos tipos las rectoras, que atraviesan el intercolumnio, suelen ceñirse a la vertical maestra externa, sin prolongación en el margen de canal y las líneas verticales llegan a los bordes superior e inferior del folio.

Tipo 1

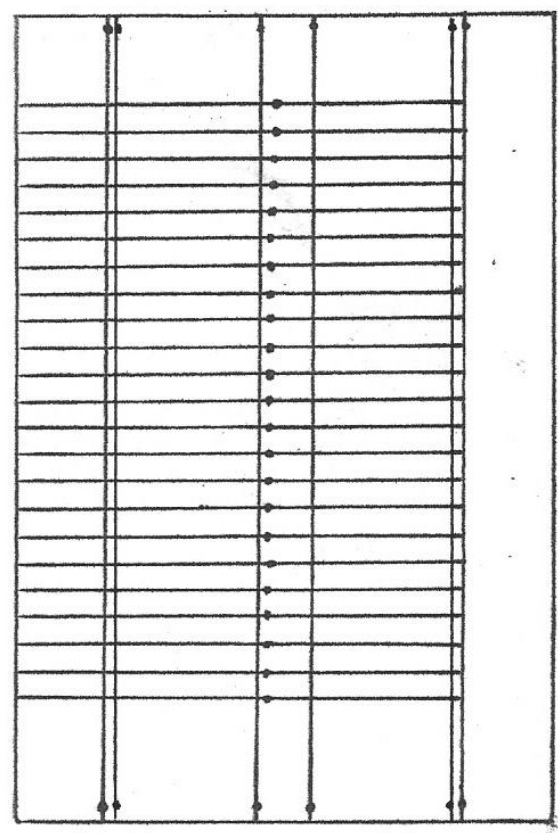

Tipo 2

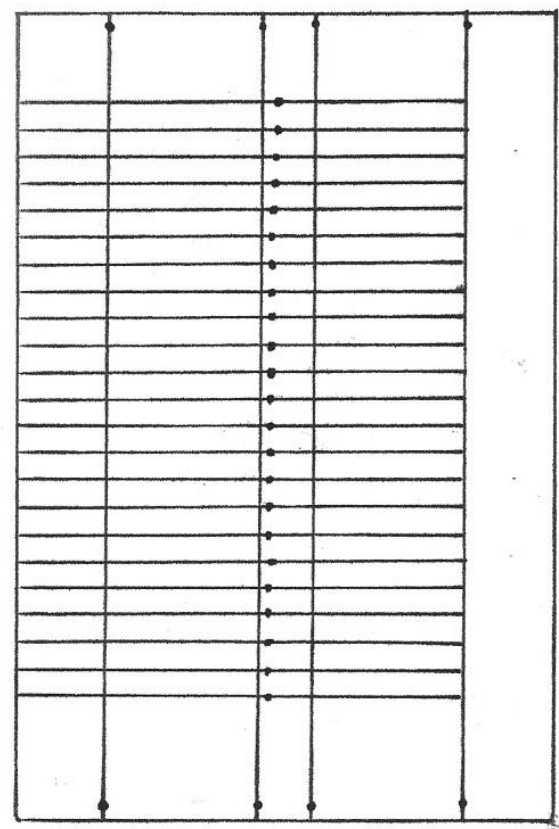

${ }^{70}$ Aparecen otros tipos puntualmente, en algunos folios sueltos destinados a recibir iluminación a toda página y en el fascículo en el que se hallan los cánones de concordancias (ff. 149-155), con páginas que se articulan mediante arcos y columnas (una línea vertical marca el centro del folio). En el f.2 se dispone una cuadrícula a punta seca que sirve de referencia al laberinto del recto. En el f.3 sólo se advierten las perforaciones necesarias para realizar los círculos en los que se introducen los rostros de los vientos que se representan en el recto y, a punta seca, se realizaron seis líneas verticales que marcan dos columnas de $67 \mathrm{~mm}$ y $59 \mathrm{~mm}$, delimitadas externamente por verticales dobles (separadas $8 \mathrm{~mm}$ y $10 \mathrm{~mm}$ respectivamente), margen de pliegue de $20 \mathrm{~mm}$, intercolumnio también de $20 \mathrm{~mm}$ y margen de canal de $50 \mathrm{~mm}$. 
Varía el número de directrices horizontales: 38 (cuadernos $2^{\circ}-3^{\circ}$ ), 47 (fascículos $26^{\circ}-27^{\circ}$ ), 50 (bifolio $100 / 107$ del cuaderno $14^{\circ}$ y cuadernos $15^{\circ}, 17^{\circ}, 18^{\circ}, 20^{\circ}$ y $21^{\circ}$ ), 51 (ff. 101-106 del cuaderno $14^{\circ}$ y fascículo $16^{\circ}$ ), 53 (cuadernos $5^{\circ}-13^{\circ}$ ), 54 (fascículo $4^{\circ}$ ) y 55 (cuadernos $22^{\circ}-25^{\circ}$ y $28^{\circ}-34^{\circ}$ ). Predominan, por tanto, los folios de 55 rectoras. Es evidente, ya a partir del tercer cuaderno, un mayor aprovechamiento del espacio, lo que no sólo se advierte en el incremento del número de renglones sin aumento relevante de la altura del cuadro de justificación -que se sitúa entre los 290 y los 299 mm-, también en la disminución del intercolumnio (35-23mm). Teniendo en cuenta, además de la morfología de los pinchazos y el número de perforaciones-guía, el tipo -1 o 2- de pautado, el número de horizontales, las dimensiones del cuadro de justificación, la anchura del intercolumnio, etc., es posible identificar a lo largo del códice más de diez configuraciones de página distintas.

Como ejemplo, sirvan las siguientes descripciones:

f. $4 \mathrm{r}: 20+6+65+35+63+6+45$ x 29+295+46 mm. Tipo 1 de pautado. Cuadro de justificación: 295x175mm. Rectrices: 38. UR: 7,972mm.

$\checkmark$ f.20r: $20+71+24+71+52 \times 21+299+50 \mathrm{~mm}$. Tipo 2 de pautado. Cuadro de justificación: 299x168mm. Rectrices: 54. UR: 5,641 mm.

$\checkmark$ f.203r: $30+70+23+66+50 \times 31+290+48 \mathrm{~mm}$. Tipo 2 de pautado. Cuadro de justificación: 290x159. Rectrices: 47. UR: 6,304 mm

$\checkmark$ f.218r: $22+70+25+65+55$ x 26+290+52 mm. Tipo 2 de pautado. Cuadro de justificación: 290x160. Rectrices: 55. UR: 5,370 mm.

$\checkmark$ f.258r: $28+71+24+65+51 \times 19+290+62 \mathrm{~mm}$. Tipo 2 de pautado. Cuadro de justificación: 290x160 mm. Rectrices: 55. UR: 5,370mm.

Para valorar adecuadamente la proporción negro/blanco en las páginas, hemos de tener en cuenta que, como se advirtió anteriormente, las dimensiones de los márgenes no son las originarias. Salvo excepciones -justificadas por el contenido, la inserción de epígrafes destacados o defectos del pergamino- coincide el número de líneas rectrices con el de renglones escritos.

\section{TINTAS Y PINTURAS.}

Un primer recorrido por los folios del manuscrito pone ya de relieve la variedad cromática. Predominan las tintas negras destinadas al texto base realizado en escritura ordinaria, el titulus currens y los reclamos. También se emplearon para la ejecución de epígrafes en escritura notoria, en iniciales decoradas y en las 
ilustraciones. Aun admitiendo el calificativo negro, más que de tinta negra propiamente dicha se trata de negra-pardusca o marrón. No existe homogeneidad en el color, no faltan las páginas con texto en tinta desvaída. Ocasionalmente, las diferencias en la composición de la mezcla se relacionan con cambios de mano en la escritura ${ }^{71}$.

La tinta roja es frecuente a lo largo del manuscrito, como recurso esencial para la identificación y articulación de los textos (numerales, encabezamientos de apartados realizados en escritura ordinaria, epígrafes en caracteres publicitarios, etc.). También se utilizó rojo en las signaturas de cuaderno (inicial $Q$, numeral, figura ornamental), en el ex libris del f.2r, en las suscripciones de los ff. 2r, 202r y 211r, en las explanationes que acompañan las imágenes a toda página del f.3r y las escenas que se disponen en cuatro registros en el f.201v. Es habitual, asimismo, su empleo -para el contorno o el relleno, según los casos- en mayúsculas destacadas secundarias. Tinta y pintura roja en varias tonalidades aparecen, asimismo, en iniciales figuradas y secundarias, en los elementos arquitectónicos de configuración de página y en las miniaturas propiamente dichas.

El verde es también un color muy presente en el ejemplar. Se destina a la escritura decorativa -caracteres publicitarios en epígrafes, iniciales secundarias, mayúsculas figuradas-, a los elementos arquitectónicos y fitomorfos que articulan la superficie de ciertas páginas y a las ilustraciones propiamente dichas. Para estos mismos usos se recurrió también al azul y al amarillo. Excepcionalmente, se realizaron en tinta azul encabezamientos en escritura ordinaria ${ }^{72}$.

\section{PUNTO DE PARTIDA.}

Son los precedentes sólo unos primeros apuntes "de campo" sobre la materialidad del Códice $6 \mathrm{y}$, sin embargo, posibilitan ya un primer acercamiento a la génesis del manuscrito y permiten explicar algunas peculiaridades en la organización de su texto y en la ubicación de ciertas ilustraciones.

Es evidente que para confeccionar el códice no se disponía en el taller de pieles de buena calidad o, al menos, no en cantidad suficiente para dar fin a la obra ${ }^{73}$. Tal

${ }^{71}$ Como se advierte en el f.43r.

${ }^{72}$ En los ff. 266r-268r y $269 \mathrm{v}-274 \mathrm{r}$.

${ }^{73}$ Hay que tener en cuenta que falta, al menos, un primer volumen de la Biblia. Si la sucesión de los folios corresponde al orden en la realización material de la obra y la mayoría de los folios destinados al códice superviviente se confeccionaron después de los componentes del primer volumen (siempre cabe la posibilidad de una ejecución simultánea con el concurso de varios artífices), 
vez debamos relacionar el mayor aprovechamiento de la superficie de las páginas que se advierte a partir del cuarto fascículo con la escasez de membranas para dar fin al proyecto ${ }^{74}$. Las tachas del soporte no se deben a las operaciones ligadas a su adecuación para la recepción de escritura, al contrario. Las tareas son las correctas pero desarrolladas sobre material defectuoso y, probablemente, la pericia de los artífices ocupados de estas labores permitió optimizar los recursos. No es posible comparar el pergamino de la Biblia con el empleado en otros ejemplares coetáneos nacidos en el mismo medio; de poder hacerlo, quizá comprobaríamos que, a pesar de sus imperfecciones, se eligió para la confección del códice el mejor disponible. En lo que concierne a las tintas y pinturas, sin embargo, no se detectan, salvo excepción, problemas de calidad. Es evidente que con un conjunto de mezclas reducido se consigue tanto una buena articulación del texto como un efecto estético más que notable. El deseo de hacer una obra bella no sólo se advierte en el colorido, la escritura armónica y la introducción de ilustraciones, también las humildes signaturas de cuaderno han recibido decoración.

La optimización de los medios materiales y el desempeño de las operaciones preparatorias para la escritura y la iluminación son consecuencia, sin duda, del trabajo de artífices expertos, tal vez los mismos que se ocuparon de labores más comprometidas como la copia de los textos y la iluminación, algo imposible de demostrar por el momento, sin un estudio pormenorizado de la escritura y de la decoración. La utilización de más de un instrumento para la ejecución del pinchado y las diferentes configuraciones de página a lo largo del volumen podrían deberse a la participación en estas tareas de varios artesanos y, probablemente también, su desarrollo en distintos tiempos. La Biblia es, sin duda, obra de un grupo, de un taller de cierta envergadura.

Además de la mise en page -tipos de pautado, número de rectrices por folio, morfología de las perforaciones-, otros aspectos de la materialidad -fasciculación, sucesión de signaturas de cuadernos, aparición de páginas total o parcialmente en blanco, etc.- indican que el orden en la realización de los folios no coincide con su posición definitiva en el códice, algo que confirma el contenido.

El volumen es muy homogéneo en fasciculación. De los treinta y cuatro cuadernos, veintinueve son cuaterniones. El recurso a un cuaderno distinto -en este caso de diez folios- al final del códice no sorprende, es una práctica habitual para

pudieron emplearse primeramente las membranas de mejor calidad, como se advierte en otros manuscritos.

${ }^{74}$ Caben, no obstante, otras explicaciones: empleo de folios preparados para otros libros, imitación del modelo, etc. 
ajustar el soporte al contenido. Tampoco es difícil explicar la presencia de otros fascículos de estructura distinta y, a través de ellos, con ayuda de las signaturas de cuaderno, establecer varias unidades en la composición del libro. El fascículo facticio de tres folios que abre el volumen, y que recoge ilustraciones de gran interés, no fue computado en la sucesión de signaturas. Se elaboró, lo que posibilita -y aconseja- su contenido, independientemente. La posición de los dos primeros folios parece la adecuada, si lo comparamos con otros manuscritos coetáneos "cruz de Oviedo" (f.1v) y laberinto (f.2r)-, pero ¿iban inicialmente destinados a esta segunda parte de la Biblia o a la primera ahora desaparecida? No podemos perder de vista que en la última página del códice (f.275v) se habían previsto, además del colofón, dos laberintos. En cuanto al tercer folio, con ilustraciones consideradas excepcionales en un manuscrito bíblico -rosa de los vientos (f.3r) y página tapiz con figuras humanas y animales en círculos (f.3v)- ¿se realizó para éste o para otro ejemplar de texto distinto fruto del mismo taller?

Las modificaciones en las signaturas de cuaderno a partir de la dispuesta en el $\mathrm{f}$. $171 \mathrm{v}$-raspado de numerales, enmiendas- nos privan de información relevante para establecer las fases en la composición del volumen y el orden de los fascículos previsto en un principio. Aun así, proporcionan algunos datos de interés al respecto. El primer fascículo del volumen, como señalé antes, no fue tenido en cuenta en la sucesión de signaturas, sin embargo, el cuaternión preparado para recibir los "cánones de concordancias" y que, sin lugar a dudas, se confeccionó también independientemente, pese a no portar este elemento de ordenación, sí fue computado $^{75}$, lo que indica que su ubicación en el códice ya era conocida. Sin embargo, hay dos cuadernos -el $27^{\circ}$ y el $28^{\circ}$ (ff. 202-217)-, carentes de signaturas, que no se consideraron en la numeración y los únicos, además, en los que el reclamo -en el f.209v- es su único elemento de ordenación. A esta particularidad pueden añadirse otras. Los pinchazos para orientar la pauta tienen morfología circular y los de los fascículos precedentes $\left(19^{\circ}-25^{\circ}\right)$ y siguientes $\left(28^{\circ}-32^{\circ}\right)$ son planos, el número de rectoras es de sólo 47 -los folios anteriores y posteriores presentan 55- y tanto la última página -f. $217 \mathrm{v}$ - como parte de la penúltima ${ }^{76}$ permanecen en blanco. Según se deduce de su materialidad, los ff. 202-217 conforman un bloque independiente, aunque nacido en el mismo contexto, cuya posición - ¿y pertenencia?- en el volumen no estaba prevista desde el comienzo. Esta individualidad revelada por la forma se ve corroborada por el fondo: las páginas recogen diversos tratados que, insertos entre el evangelio de Juan y

\footnotetext{
${ }^{75}$ La signatura del cuaderno anterior -f.148v- es $Q X V I I$ y la del fascículo siguiente, en el f.163v, Q XVIIII.

${ }^{76}$ El final de la segunda columna.
} 
Hechos, rompen la sucesión de la Sagrada Escritura. Hay otro aspecto que confiere a este conjunto especial relieve. En sus páginas se encuentran las representaciones simbólicas de los evangelistas que sorprenden por su configuración a los historiadores del Arte y cuatro de las cinco suscripciones del "diácono Juan". La primera suscripción de Iohannes ${ }^{77}$ se ubica en el f.91r, página en su mayor parte en blanco que cierra otra unidad del códice, ésta si considerada en las signaturas y, en lo que se refiere al contenido, destinada a guardar los libros proféticos.

La composición de los cuadernos $17^{\circ}$ y $18^{\circ}$ (ff. 124-148), de doce y trece folios respectivamente, se debe también a su condición de "fascículos finales", no del códice en su totalidad, sino de la unidad que comprende los ff. 92-148, antes de la introducción del cuaternión -autónomo- dedicado a los cánones.

Para el cuaderno $25^{\circ}$ (ff. 195-201), también irregular, aún no he encontrado una explicación convincente. Consta de siete folios, se rompe en él la Ley de Gregory y carece de signatura (se ha realizado una figura fitomorfa, sin inicial ni numeral en su interior). Debido a las correcciones en los numerales de las signaturas siguientes no puede demostrarse que fuese tenido en cuenta en el cómputo. Ignoro, por el momento, si la modificación responde a la inserción de las cuatro ilustraciones neotestamentarias que se hallan en el f.201v -no incluidas para ocupar espacio sobrante puesto que, precisamente, la escritura del folio (r-v) va disminuyendo progresivamente de tamaño para acomodar el texto que ha de trasladarse al menor espacio disponible ${ }^{78}$ - o para cerrar otra unidad en el volumen dedicada a los textos evangélicos y sus piezas introductorias.

La resolución de ésta y otras incógnitas habrá de esperar a nuevas "campañas".

\footnotetext{
${ }^{77}$ Me refiero a suscripciones originarias. En el f.101r -en el texto de la Historia de san Froilán (véase la nota 15)- aparece una suscripción contemporánea a esta adición.

${ }^{78} \mathrm{Sin}$ modificación en el pautado, la letra se va haciendo más pequeña. Al comienzo del f.201r el cuerpo de los caracteres mide $2 \mathrm{~mm}$ de altura y en el f.201v ya sólo $1 \mathrm{~mm}$.
} 